\title{
Monitoramento da atividade de lesões iniciais de cárie em alunos de uma escola do município de Maceió-AL após tratamento preventivo conservador
}

\author{
Monitoring the activity of initial injuries of caries in students in a school in the municipality \\ of Maceió-AL after conservatory preventive treatment
}

Monitoreo de la actividad de lesiones iniciales de caries en alumnos de una escuela del municipio de Maceió-AL tras tratamiento preventivo conservador

Izabel Cristina Gomes de Mendonça ${ }^{1}$, Diana Xavier de Barros Padilha ${ }^{2}$, Roberta Alexandre Lima ${ }^{1}$, Maria Carolyna Ferreira Alapenha ${ }^{3}$, Perolina Maria Lima de Medeiros ${ }^{4}$, Thaís de Amorim Camerino ${ }^{1 *}$.

\section{RESUMO}

Objetivo: O objetivo do trabalho foi observar a atividade de mancha branca de cáries e os índices de higiene bucal (cárie, biofilme dental e sangramento gengival), monitorando a resposta ao tratamento de lesões de mancha branca ativa e inativa submetidas a tratamento preventivo e conservador a longo prazo. Método: Trata-se de um estudo transversal conduzido a partir da análise de 331 prontuários de pacientes de uma escola do município de Maceió-AL, no período de 2011 a 2015. Resultado: Foi considerado que 83,33\% das manchas brancas ativas inativaram na revisão em relação a consulta inicial; $13,85 \%$ das manchas brancas inativas diagnosticadas na primeira consulta cavitaram e os índices indicativos de saúde bucal diminuíram significativamente. Conclusão: Assim, os resultados desta pesquisa estão em consonância com as novas diretrizes da Dentística e demonstram que é possível prevenir e tratar a doença cárie com intervenções simples e conservadoras.

Palavras-Chave: Cárie Dentária, Flúor, Odontologia Preventiva.

\begin{abstract}
Objectiv: The objective of the study was to observe the white spot caries activity and the indexes of oral hygiene (caries, dental biofilm and gingival bleeding), monitoring the response to treatment of active and inactive white spot lesions submitted to long term preventive and conservative treatment deadline. Method: This is a cross-sectional study based on the analysis of 331 patient records of a school in the municipality of Maceió-AL, from 2011 to 2015 . Results: It was considered that $83.33 \%$ of the active white spots inactivated the revision in relation to the initial consultation; $13.85 \%$ of the inactive white spots diagnosed at the first consultation cavitated and the indicative indices of oral health decreased significantly. Conclusion:Thus, the results of this research are in line with the new guidelines of Dentistry and demonstrate that it is possible to prevent and treat a disease with simple and conservative actions.
\end{abstract}

Palavras-Chave: Dental Caries, Fluorine, Preventive Dentistry.

\section{RESUMEN}

Meta: El objetivo del trabajo fue observar la actividad de mancha blanca de caries y los índices de higiene bucal (caries, biofilm dental y sangramiento gingival), monitoreando la respuesta al tratamiento de lesiones de mancha blanca activa e inactiva sometidas a tratamiento preventivo y conservador a largo plazo plazo. Método: Se trata de un estudio transversal conducido a partir del análisis de 331 prontuarios de pacientes de una escuela del municipio de Maceió-AL, en el período de 2011 a 2015. Resultados: Se consideró que el

${ }^{1}$ Centro Universitário Cesmac - Maceió/AL.

${ }^{2}$ Universidade de Coimbra - Portugal.

${ }^{3}$ Faculdade São Leopoldo Mandic - Campinas/SP.

${ }^{4}$ Serviço Social do Comécio - SESC/AL. *E-mail: tcamerino@hotmail.com

SUBMETIDO EM: 5/2018

ACEITO EM: 6/2018

PUBLICADO EM: $\mathbf{3 / 2 0 1 9}$

REAS/EJCH | Vol.Sup.20 | e584 | DOI: https://doi.org/10.25248/reas.e584.2019 Página 1 de 10 
$83,33 \%$ de las manchas blancas activas inactivaron en la revisión en relación la consulta inicial; El 13,85\% de las manchas blancas inactivas diagnosticadas en la primera consulta cavitaron y los índices indicativos de salud bucal disminuyeron significativamente. Conclusión:Así, los resultados de esta investigación están en consonancia con las nuevas directrices de la Dentística y demuestran que es posible prevenir y tratar la enfermedad caries con intervenciones simples y conservadoras.

Palabras Clave: Caries dentales, El fluoruro, Odontología Preventiva.

\section{INTRODUÇÃO}

O maior conhecimento dos mecanismos de instalação e desenvolvimento da cárie, bem como a evolução tecnológica alcançada pelos materiais restauradores no quesito adesividade e liberação de flúor, indubitavelmente contribuíram para uma mudança significativa no tratamento da doença, o qual passou por profundas modificações ao longo do tempo, permitindo sua prevenção e paralização (PERES et al., 2001; AUBUCHAIM, 2001).

No momento em que foi demonstrado que tratar as sequelas da cárie não impediam a reinstalação da doença e tampouco contribuíam para uma efetiva diminuição na prevalência da mesma, uma nova filosofia de tratamento, ancorada em um diagnóstico preciso e no controle efetivo dos mecanismos da doença que a originam, surgiu com resultados animadores (CARNEIRO, 2003).

É sabido que a cárie é uma manifestação clínica de uma infecção bacteriana (LIMA, 2007) que causa prejuízo à qualidade de vida, gerando dor e acarretando consequências negativas na mastigação, fonação, estética e na interação social de seus portadores (CONCEIÇÃO e LEITE, 2007). Em seus aspectos microbiológicos, caracteriza-se pela dissolução química dos sais de cálcio do dente pelo ácido lático, acompanhada pela decomposição da matriz orgânica, ou corpo gelatinoso, que, na dentina, ocorre após a dissolução dos sais de cálcio. É causada por bactérias que colonizam a superfície do dente e produzem ácidos como resultados do metabolismo dos carboidratos (FEJERSKOV, KIDD, 2005).

O primeiro sinal clínico passível de detecção a olho nu denomina-se lesão de mancha branca ativa, na qual o esmalte mostrar-se opaco e sem translucidez, estão geralmente situadas onde existe acúmulo de biofilme, e na ausência de tratamento progredirá até a destruição total da coroa dentária. Já as lesões de esmalte inativas apresentam superfície lisa, brilhante e geralmente não existe biofilme associado (GUIRADO et al., 2006).

A abordagem convencional usada no tratamento das lesões de cárie baseia-se em procedimentos clínicos invasivos para restabelecer a integridade da superfície dental e devolver a função do mesmo através de uma restauração (HESSE et al., 2014). Porém, condutas minimamente invasivas baseadas na desorganização mecânica e química do biofilme dental, como a administração de fluoretos e antimicrobianos (SOARES, VALENCA, 2001) por profissionais, estão sendo preferidas no tratamento de lesões iniciais, tanto pela eficácia comprovada como também pela preservação da estrutura dental (SOARES, VALENCA, 2003).

O emprego do tratamento não operatório propõe interromper a atividade das lesões, sobretudo aquelas situadas em superfícies livres e lisas, conservando os tecidos duros circundantes (KIDD, NYVAB, 2011).

O conceito de prevenção da doença cárie tem sido amplamente discutido uma vez que as medidas utilizadas podem causar interferência no processo de desmineralização-remineralização do elemento dentário, induzindo a estabilização ou a redução da velocidade de progressão da carie (COSTA, 2009).

Segundo Maltz et al. (2010), a prevenção e o tratamento da cárie dentária estão relacionados a fatores comportamentais, incluindo hábitos de higiene bucal e alimentares, relacionados a muitas doenças crônicas, e concluem que ações, como as políticas de alimentação, saúde e higiene em geral (incluindo higiene bucal), entre outros devem ser integradas às estratégias de base da promoção da saúde, associadas ao tratamento da lesão cariosa quando da sua presença. 
A evolução filosófica na odontologia é o resultado de experiências que levaram a novas evidências comportamentais da ciência, refletindo em necessidades de mudanças e práticas da Dentística (FEJERSKOV, KIDD, 2005). Durante muito tempo verificou-se a importância da prevenção como a melhor forma de evitar o início ou progressão de doenças bucais. A odontologia preventiva tem como objetivo traçar medidas de controle da doença cárie as quais incluem influenciar a formação e o desenvolvimento do biofilme e/ou modificar a cinética da dissolução das apatitas (DANTAS, 2015ç ROZIER, 2001).

Esse modelo de prevenção é adotado nas clínicas odontológicas do SESC - Serviço Social do Comércio, que é uma entidade privada que tem como missão a promoção do bem-estar dos comerciários e seus dependentes, por meio de ações desenvolvidas nas áreas de educação, saúde, cultura, lazer e assistência, onde os serviços odontológicos são organizados atendendo aos preceitos da promoção de saúde bucal e estruturado na atenção primária a saúde, que se traduz em cuidados básicos que valorizam as ações de promoção, prevenção e assistência individual e manutenção da saúde e prevenção de seus agravos (SESC, 2015).

O SESC Alagoas desenvolve um projeto denominado Estação Sorriso que tem como público alvo os alunos matriculados na Educação Infantil e Ensino Fundamental da Unidade SESC JARAGUÁ. Este projeto privilegia ações educativas (escovação supervisionada quinzenal, escovação diária, aplicações tópicas de flúor, atividades educativas com os grupos) e tratamento curativo na clínica odontológica quando este se faz necessário (SESC, 2015).

O objetivo da presente pesquisa foi avaliar o comportamento clínico das lesões de mancha branca ativa e inativa submetidos a tratamento preventivo e conservador a longo prazo.

\section{METODOLOGIA}

Consiste de um estudo observacional retrospectivo que foi desenvolvido na clínica odontológica de uma instituição prestadora de serviço, privada, com os alunos da escola da citada instituição que participaram do projeto Estação Sorriso. A pesquisa foi aprovada pelo Comitê de Ética e Ensino em Pesquisa (COEPE) do Centro Universitário Cesmac sob o parecer no 1431517

Os prontuários deste estudo foram selecionados dentre os indivíduos que participaram do projeto Estação Sorriso, de ambos os gêneros, que receberam tratamento odontológico preventivo/conservador. Essa seleção foi feita uma vez na semana, por dias alternados, entre os meses janeiro e junho de 2016 em dias da semana alternados na Clínica Odontológica do SESC, onde os prontuários eram cedidos pela cirurgiã-dentista responsável pelo projeto.

Ao final da análise de 331 prontuários, apenas 107 foram julgados aptos para a análise estatística dos dados, seguindo os critérios de inclusão da pesquisa (alunos matriculados na Educação infantil e Ensino Fundamental da Escola SESC Jaraguá e que frequentam a clínica odontológica da Instituição, indivíduos na faixa etária de 03 a 11 anos de idade, participar do projeto denominado Estação Sorriso e ser atendido por um único odontólogo responsável pelo projeto, indivíduos atendidos no período compreendido entre 2011 a 2015 e indivíduos com histórico de lesão de mancha branca ativa (cavitada ou não), lesões inativas e também os livres de cárie).

Não houve a necessidade do preenchimento do Termo de Consentimento Livre e Esclarecido por se tratar de um estudo retrospectivo e observacional. As informações dos prontuários retiradas para esta pesquisa foram em dois momentos: 1) Dados coletados iniciais (1 ${ }^{\text {a }}$ consulta) e, 2) Dados coletados nas consultas de retorno.

Para análise dos dados foram obtidas distribuições absolutas, percentuais e as medidas estatísticas: média, mediana, desvio padrão, valor mínimo e valor máximo (Técnicas de estatística descritiva) e foram utilizadas Técnicas de estatística inferencial através dos testes de Wilcoxon para dados pareados quando os dados foram analisados como variáveis numéricas ou Mc-Nemar quando os dados foram analisados em forma de categoria. 
A margem de erro utilizada nas decisões dos testes estatísticos foi de $5 \%$. Os dados foram digitados na planilha EXCEL e o programa utilizado para obtenção dos cálculos estatísticos foi o SPSS (Statistical Package for the Social Sciences) na versão 23.

\section{RESULTADOS}

A idade dos pesquisados variou de 3 a 10 anos, teve média de 6,25 anos, desvio padrão de 1,56 anos e mediana de 6,00 anos. Na Tabela 1 se apresenta os resultados das características faixa etária e sexo dos pacientes pesquisados. Esta tabela mostra que as duas faixas etárias mais frequentes foram: 3 a 5 anos e 6 a 7 cada uma com $38,9 \%$ da amostra e os $22,1 \%$ restantes tinham 8 a 10 anos; um pouco mais da metade $(53,7 \%)$ era do sexo feminino.

Na Tabela 2 se apresenta as estatísticas média, desvio padrão, mediana, quartil 1 (Q1) e quartil 3 (Q3) para as variáveis número de dentes de dentes hígidos, número de dentes cariados, manchas brancas ativas (MBA), manchas brancas inativas, índice de placas visível (IPV) e índice de sangramento gengival (ISG) em cada uma das avaliações. Desta tabela se destaca que: a média do número de dentes hígidos teve um pequeno aumento de 16,62 para 16,72 dentes e a mediana variou de 17,00 para 18,00 dentes e para a margem de erro fixada (5\%) não se comprova diferença significativa entre as duas avaliações $(p>0,05)$; as médias das variáveis: número de dentes cariados, MBA, IPV e ISG reduziram da primeira consulta para a avaliação da revisão enquanto que o número de $\mathrm{MBI}$ aumentou de 1,52 para 2,08 , diferenças estas que se mostraram significativas $(p<0,05)$. A variabilidade expressa através do desvio padrão foi bastante elevada exceto no número de dentes hígidos desde que as referidas medidas foram próximas ou superiores as correspondentes médias.

Tabela 1 - Distribuição dos pesquisados segundo a faixa etária e sexo

\begin{tabular}{lll}
\hline Variável & $\mathbf{n}$ & $\%$ \\
\hline TOTAL & 95 & 100,0 \\
Faixa etária (anos) & & \\
3 a 5 & 37 & 38,9 \\
6 a 7 & 37 & 38,9 \\
8 a 10 & 21 & 22,1 \\
Sexo & & \\
Masculino & 44 & 46,3 \\
Feminino & 51 & 53,7 \\
\hline
\end{tabular}

Fonte: Dados da Pesquisa.

$\mathrm{Na}$ Tabela 3 se analisa número de dentes de dentes hígidos, número de dentes cariados, manchas brancas ativas (MBA), manchas brancas inativas, índice de placas visível (IPV) e índice de sangramento gengival (ISG) categorizados em cada uma das avaliações. Desta tabela com exceção do número de dentes hígidos se comprova diferença significativa $(p<0,05)$ entre as duas consultas e para as variáveis com diferença significativa se destaca que: a maioria em cada avaliação não tinha dentes com cárie, entretanto este percentual foi mais elevado na segunda avaliação do que na revisão $(84,2 \%$ x 53,7\%) e os percentuais com 1 a 4 dentes cariados e 5 a 10 foram correspondentemente mais elevados na primeira consulta (37,9\% x $14,7 \%$ com a 1 a 4 dentes cariados e $8,4 \%$ x $1,1 \%$ com 5 a 10 dentes cariados); a maioria não apresentava manchas brancas ativas, sendo este percentual mais elevado na revisão $(90,5 \%$ x $64,2 \%)$; a maioria tinha 1 a 3 manchas brancas inativas e o percentual com ausência de manchas brancas inativas foi mais elevado na primeira consulta $(22,1 \%)$, enquanto que os percentuais com 1 a 3 ou 4 a $8 \mathrm{MBI}$ foram correspondentemente 
mais elevados na revisão do que na primeira consulta $(82,1 \%$ x 66,3\% com 1 a 3 e $14,7 \%$ x 11,6\%); a maioria das crianças tinha ausência de faces com placas visíveis, sendo que o referido percentual foi mais elevado na revisão $(78,9 \% \times 51,6 \%)$ enquanto que os percentuais com 1 a 4 faces e 5 a 19 foram correspondentemente mais elevados na primeira consulta do que na revisão $(25,3 \% \times 7,4 \%$ com 1 a 4 faces e $23,2 \% \times 13,7 \%$ com 5 a 19 faces); a maioria não apresenta faces com sangramento sendo $85,8 \%$ na primeira consulta e $98,9 \%$ na revisão (Gráfico 1).

Tabela 2 - Dados estatísticos do número e dentes hígidos, cariados, MBA, MBI, IPV e ISG segundo a consulta (avaliação).

\begin{tabular}{|c|c|c|c|c|}
\hline Variável & Estatísticas & $1^{\circ}$ consulta & Revisão & Valor de $p$ \\
\hline \multirow[t]{5}{*}{$\mathrm{N}^{\circ}$ de dentes hígidos } & Média & 16,62 & 16,72 & $\mathrm{p}^{(1)}=0,679$ \\
\hline & Desvio padrão & 4,11 & 3,80 & \\
\hline & Mediana & 17,00 & 18,00 & \\
\hline & Q1 & 14,00 & 15,00 & \\
\hline & Q3 & 19,00 & 19,00 & \\
\hline \multirow[t]{5}{*}{$\mathbf{N}^{\circ}$ de dentes cariados } & Média & 1,25 & 0,37 & $\mathrm{p}^{(1)}<0,001^{*}$ \\
\hline & Desvio padrão & 1,93 & 1,01 & \\
\hline & Mediana & 0,00 & 0,00 & \\
\hline & Q1 & 0,00 & 0,00 & \\
\hline & Q3 & 2,00 & 0,00 & \\
\hline \multirow[t]{5}{*}{ MBA } & Média \pm DP & 0,96 & 0,17 & $\mathrm{p}^{(1)}<0,001^{*}$ \\
\hline & Desvio padrão & 1,78 & 0,58 & \\
\hline & Mediana & 0,00 & 0,00 & \\
\hline & Q1 & 0,00 & 0,00 & \\
\hline & Q3 & 1,00 & 0,00 & \\
\hline \multirow[t]{5}{*}{ MBI } & Média \pm DP & 1,52 & 2,08 & $\mathrm{p}^{(1)}<0,001^{*}$ \\
\hline & Desvio padrão & 1,46 & 1,56 & \\
\hline & Mediana & 1,00 & 2,00 & \\
\hline & Q1 & 1,00 & 1,00 & \\
\hline & Q3 & 2,00 & 2,00 & \\
\hline \multirow[t]{5}{*}{ IPV } & Média \pm DP & 2,73 & 1,52 & $\mathrm{p}^{(1)}=0,004^{*}$ \\
\hline & Desvio padrão & 4,17 & 3,56 & \\
\hline & Mediana & 0,00 & 0,00 & \\
\hline & Q1 & 0,00 & 0,00 & \\
\hline & Q3 & 4,00 & 0,00 & \\
\hline \multirow[t]{5}{*}{ ISG } & Média \pm DP & 0,44 & 0,02 & $\mathrm{p}^{(1)}<0,001^{*}$ \\
\hline & Desvio padrão & 1,30 & 0,21 & \\
\hline & Mediana & 0,00 & 0,00 & \\
\hline & Q1 & 0,00 & 0,00 & \\
\hline & Q3 & 0,00 & 0,00 & \\
\hline
\end{tabular}

(*): Diferença significativa ao nível de 5,0\%. (1): Através do teste de Wilcoxon para dados pareados. Fonte: Dados da Pesquisa. 
Tabela 3 - Avaliação do número de: dentes hígidos, cariados, MBA, MBI, IPV e ISG segundo a consulta (avaliação).

\begin{tabular}{|c|c|c|c|c|c|}
\hline \multirow[t]{2}{*}{ Variável } & \multicolumn{2}{|c|}{$1^{\circ}$ consulta } & \multicolumn{2}{|c|}{ Revisão } & \multirow[t]{2}{*}{ Valor de $p$} \\
\hline & $\mathbf{N}$ & $\%$ & $\mathbf{n}$ & $\%$ & \\
\hline TOTAL & 95 & 100,0 & 95 & 100,0 & \\
\hline \multicolumn{6}{|c|}{$N^{\circ}$ de dentes hígidos } \\
\hline 3 a 12 & 16 & 16,8 & 14 & 14,7 & $p^{(1)}=0,607$ \\
\hline 13 a 18 & 46 & 48,4 & 48 & 50,5 & \\
\hline 19 a 23 & 33 & 34,7 & 33 & 34,7 & \\
\hline \multicolumn{6}{|c|}{$\mathbf{N}^{\circ}$ de dentes cariados } \\
\hline 0 & 51 & 53,7 & 80 & 84,2 & $\mathrm{p}^{(1)}<0,001^{*}$ \\
\hline 1 a 4 & 36 & 37,9 & 14 & 14,7 & \\
\hline \multicolumn{6}{|l|}{ MBA } \\
\hline Ausente & 61 & 64,2 & 86 & 90,5 & $\mathrm{p}^{(1)}<0,001^{*}$ \\
\hline Presente (1 a 9) & 34 & 35,8 & 9 & 9,5 & \\
\hline \multicolumn{6}{|l|}{ MBI } \\
\hline Ausente & 21 & 22,1 & 3 & 3,2 & $\mathrm{p}^{(1)}=0,001^{*}$ \\
\hline 1 a 3 & 63 & 66,3 & 78 & 82.1 & \\
\hline 4 a 8 & 11 & 11,6 & 14 & 14,7 & \\
\hline \multicolumn{6}{|l|}{ IPV } \\
\hline Nenhuma & 49 & 51,6 & 75 & 78,9 & $\mathrm{p}^{(1)}<0,001^{*}$ \\
\hline 1 a 4 & 24 & 25,3 & 7 & 7,4 & \\
\hline 5 ou 19 & 22 & 23,2 & 13 & 13,7 & \\
\hline \multicolumn{6}{|l|}{ ISG } \\
\hline Nenhuma & 81 & 85,3 & 94 & 98,9 & $\mathrm{p}^{(1)}<0,001^{*}$ \\
\hline Um ou mais ( 1 a 6 ) & 14 & 14,7 & 1 & 1,1 & \\
\hline
\end{tabular}

$\left(^{\star}\right)$ : Diferença significativa ao nível de 5,0\%. (1): Através do teste McNemar.

Fonte: Dados da Pesquisa.

Tabela 4 - Dados estatísticos das cáries ativas na primeira consulta e dos resultados na revisão.

\begin{tabular}{llll}
\hline Estatísticas & $\mathbf{1}^{\circ}$ Consulta ativas & Revisão permaneceram ativas & Inativaram \\
\hline Média & 0,96 & 0,17 & 0,79 \\
Desvio padrão & 1,78 & 0,58 & 1,53 \\
Mediana & 0,00 & 0,00 & 0,00 \\
Q1 & 0,00 & 0,00 & 0,00 \\
Q3 & 1,00 & 0,00 & 1,00 \\
\hline
\end{tabular}

Fonte: Dados da Pesquisa 
Gráfico 1 - Indicadores de saúde bucal antes e após tratamento preventivo conserva.

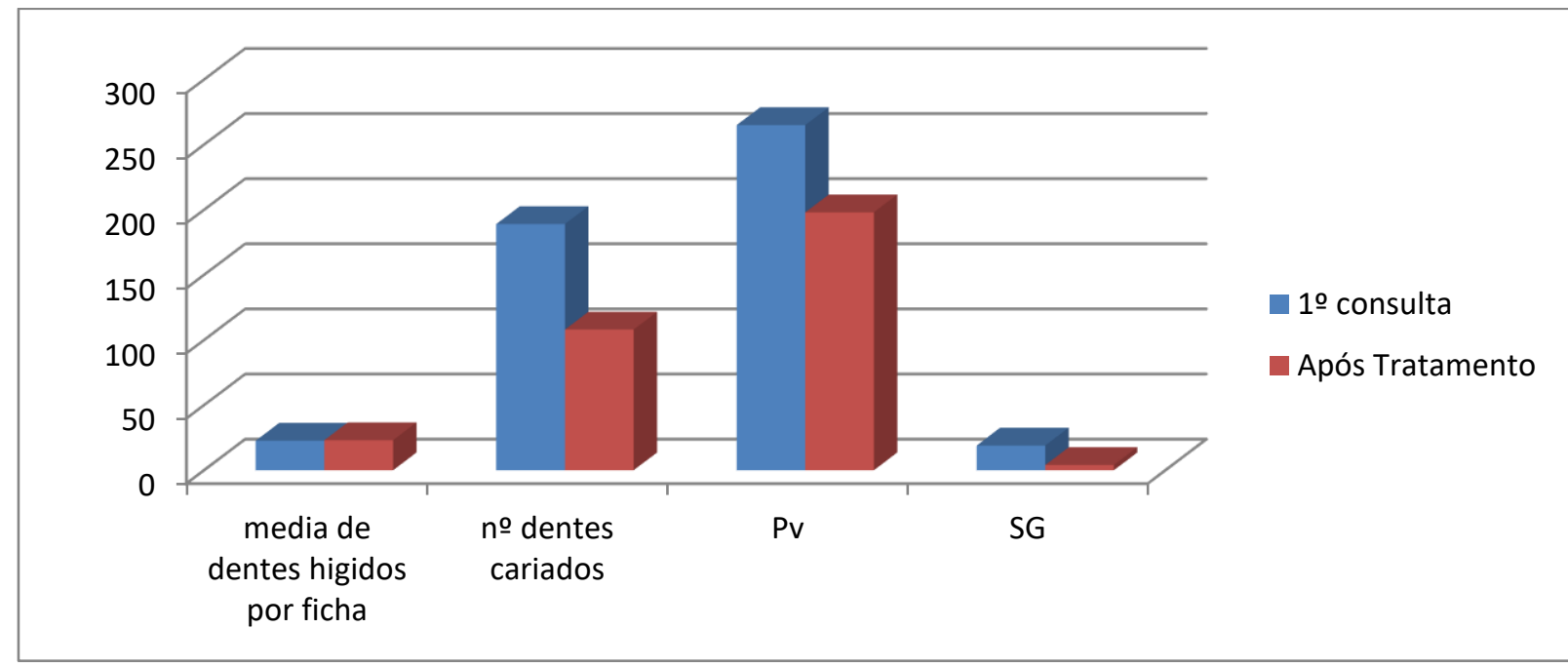

Fonte: Dados da Pesquisa.

Gráfico 2 - Indicadores do monitoramento da MBA.

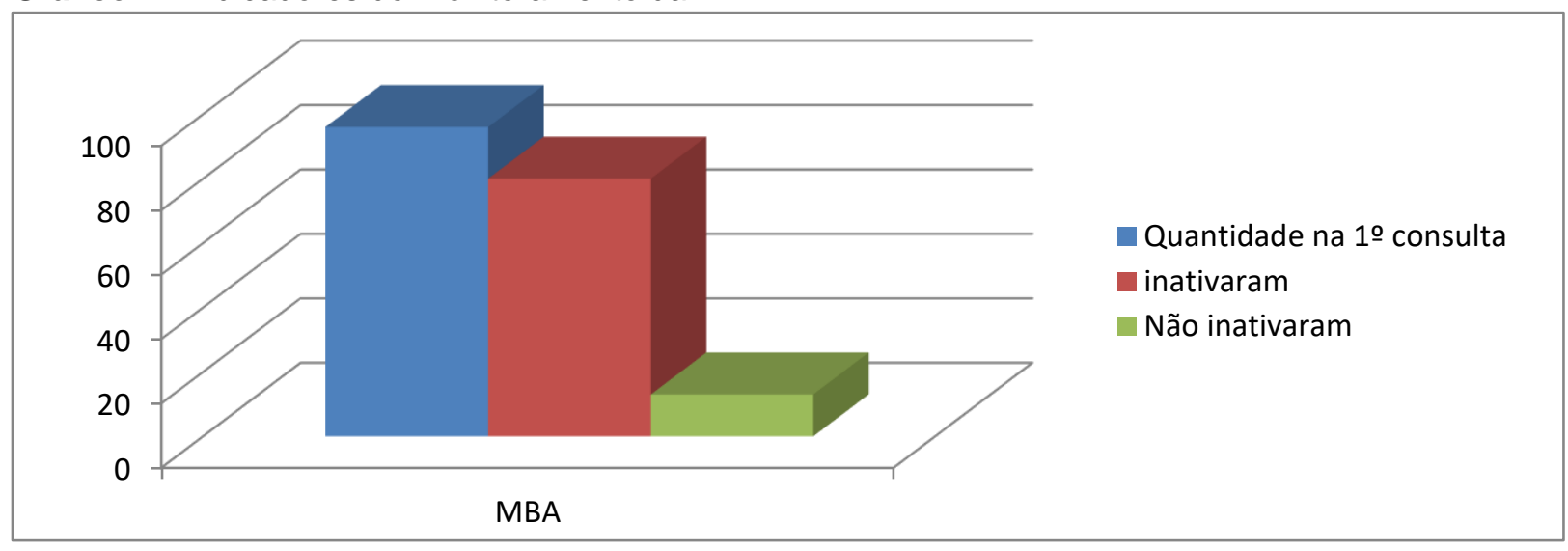

Fonte: Dados da Pesquisa.

Tabela 5 - Estatísticas do número de cáries inativas na primeira consulta e na segunda consulta.

\begin{tabular}{llcccc}
\hline \multicolumn{1}{c}{ Estatísticas } & $\begin{array}{c}\mathbf{1}^{\circ} \text { consulta } \\
\text { inativas }\end{array}$ & $\begin{array}{c}\text { Revisão } \\
\text { cavitaram }\end{array}$ & $\begin{array}{c}\text { Cáries ativas que } \\
\text { inativaram }\end{array}$ & $\begin{array}{c}\text { Permaneceram } \\
\text { inativas }\end{array}$ & $\begin{array}{c}\text { Total de } \\
\text { inativas }\end{array}$ \\
\hline Média & 1,52 & 0,22 & 0,79 & 1,30 & 2,09 \\
Desvio padrão & 1,46 & 0,73 & 1,53 & 1,25 & 1,56 \\
Mediana & 1,00 & 0,00 & 0,00 & 1,00 & 2,00 \\
Q1 & 1,00 & 0,00 & 0,00 & 0,00 & 1,00 \\
Q3 & 2,00 & 0,00 & 1,00 & 2,00 & 2,00
\end{tabular}

(1): o total de cáries inativas na revisão corresponde as que permaneceram ativas menos as que cavitaram mais as que eram ativas e inativaram ou então total das cáries que inativaram mais as que permaneceram inativas. Fonte: Dados da Pesquisa. 


\section{DISCUSSÃO}

A doença cárie tem etiologia multifatorial, seu primeiro sinal clínico, detectável a olho nu, chama-se lesão de mancha branca ativa. Que apresenta o esmalte em sua superfície sem translucidez, esbranquiçado e opaco, e associado ao acumulo de biofilme na região, que é o responsável por mediar a desmineralização dos tecidos dentários deixando uma superfície rugosa a sondagem (FERJERSKOV, 2005; FONTANA, 2009).

A abordagem da mancha branca de cárie na literatura e sua atividade provaram-se reduzidas. Porém, o estágio mais inicial da lesão tem uma grande relevância clínica, já que a mesma é passível de reversão, o que resultaria em um melhor prognóstico para a doença carie. Ainda que desde os anos 50 foram citadas nos exames clínicos (PROJETO SB BRASIL, 2010) e em pesquisas clinicas (BROADBENT, 2008) tradicionalmente, os estudos epidemiológicos da cárie dentária aferem a doença em seu estágio mais avançado, em detrimento da avaliação da sua primeira expressão clinica visível, a lesão branca de cárie (CAMPOS, 2001; SKEIE, 2006).

Na presente pesquisa, bem como na literatura consultada, nenhuma relação foi estabelecida entre o sexo e a idade dos sujeitos com o comportamento da doença ao tratamento. Porém, os índices que informam os níveis de higiene bucal (dentes hígidos, dentes cariados, percentual de sangramento gengival e percentual de placa dentária visível) da amostra estudada demonstraram interferir na atividade da mancha branca de carie. Esses índices são fortes indicadores do surgimento e progressão da doença carie (TAGLIAFERRO et al, 2008; FONTANA, ZERO, 2006).

Foi possível notar que após o tratamento preventivo não invasivo, os índices de higiene bucal (número de dentes hígidos, número de dentes cariados e índice de sangramento visível) melhoraram em comparação aos dados da primeira visita odontológica (Gráfico 1). O tratamento preventivo realizado nos participantes do projeto consiste na escovação supervisionada quinzenal e na administração semestral de flúor gel com $2 \%$ de fluoreto de sódio além de atividades lúdicas que visam incutir nas crianças a importância do cuidado com a saúde bucal. Segundo Aspiras et al (2010), o controle do biofilme é um dos principais fatores na redução do índice de cárie bem como da gengivite e do consequente sangramento gengival.

Mostrando que há evidência concreta da efetividade da instrução de higiene oral, inclusão dos dentifrícios fluoretados na escovação e no desarranjo do biofilme no controle da instalação e da atividade de lesões de cariosas (TENUTA e CURY, 2005). Tornando a análise das condições e práticas de higiene bucal fundamentais e justificáveis nesta pesquisa (FONTANA, 2009).

A escovação associada à aplicação de flúor aumenta significativamente a proteção do dente contra o ataque ácido e a colonização bacteriana visto que é capaz de promover a remineralização de lesões de cárie iniciais e sua ação antimicrobiana reduz a colonização de bactérias acidogênicas na placa (CAMPOS, 2011; ASPIRAS, 2015).

O flúor também promove a redução do pH crítico (CURY, 2016) e aumenta a resistência do esmalte à ação ácida, diminuindo a desmineralização do mesmo (BYEON, 2016; KANDUTI, 2016).

Fato comprovado pelos resultados da pesquisa com dentifrícios fluoretados desenvolvido por Damle et al (2016), ao final do trabalho os pesquisadores concluíram que os cremes dentais com flúor aumentam a dureza e diminui a perda de minerais da superfície do esmalte em comparação com dentifrícios não fluoretados.

Com relação ao monitoramento da atividade das manchas brancas ativas e inativas de cárie foi possível observar uma melhora nos resultados obtidos após o tratamento. As manchas brancas ativas (MBA) (Gráfico 2) apresentaram uma taxa de inativação de $83,33 \%$, onde da média total de manchas brancas ativas na primeira consulta 0,79 inativaram e 0,17 permaneceram ativas após o tratamento (Tabela 4).

Resultado oposto ao encontrado por Mendes (2015), em sua pesquisa, onde $55 \%$ das lesões ativas evoluíram para um estágio mais avançado da doença cárie. A diferença nos resultados se justifica pelo fato de que a população do referido estudo pertencia a uma classe menos favorecida da sociedade sem acesso tanto a medidas preventivas como a serviços de saúde de qualidade, ao passo que os sujeitos desta pesquisa 
recebiam assistência odontológica e eram monitorados periodicamente por um profissional do projeto no qual estavam inseridos.

Diversos estudos têm evidenciado a influência dos fatores socioeconômicos em uma relação diretamente proporcional com a prevalência da carie dentaria (RHIS, 2005). Em sua pesquisa, Rihs et al (2005) ratificaram essa relação, constatando que os escolares com nível socioeconômico mais elevado apresentaram melhores condições de saúde bucal.

Melhorias na condição de saúde, na qualidade de vida e, por conseguinte no resgate de sua cidadania são consequências esperadas de programas educacionais em saúde (MOIMAZ, 1992). Segundo Pauleto et. al, (2004) isso se justifica pela participação mais ativa nas ações bem como por maior acesso a informação. Desta forma a assistência odontológica do SESC vai além recuperação e reabilitação de doenças, incluindo ações de caráter de promoção de saúde tendo em vista a diminuição ou o retardamento de novas ocorrências.

As manchas brancas inativas (MBI) (Tabela 5) responderam positivamente ao tratamento preventivo conservador, onde apenas 13,85\% delas apresentaram-se cavitadas nas consultas subsequentes. Esses achados são corroborados pelos trabalhos com remineralização de manchas brancas desenvolvidos por Beyon et al. (2016), Restrepo et al (2016), WANG et al (2016) e Lijima (2008).

Estes autores compartilham da premissa de que é possível remineralizar o tecido dentário após tratamento conservador. De acordo com Lijima (2008), as lesões de mancha branca ativa podem ser remineralizadas com a adoção de procedimentos preventivos simples como escovação profissional e aplicação de flúor.

Os resultados da presente pesquisa confirmam a eficácia e a importância da adoção de estratégias não invasivas na recuperação dos tecidos duros do dente, bem como no restabelecimento da integridade da superfície dental sem a necessidade da aplicação de um material restaurador que não substitui a contento as características biofísicas do esmalte e da dentina.

\section{CONCLUSÃO}

A observação, por um período de quatro anos, do tratamento preventivo realizado nas crianças que participaram do projeto Estação Sorriso mostrou uma diminuição significativa nos índices indicativos de saúde bucal (cárie e sangramento gengival), uma taxa de inativação de $83,33 \%$ das manchas brancas ativas (MBA) em relação a consulta inicial e que uma taxa de apenas $13,85 \%$ das manchas brancas inativas (MBI) diagnosticadas na primeira consulta cavitaram.

\section{REFERÊNCIAS}

1. ABUCHAIM C. Abordagem científica e clínica do selamento de lesões de cárie em superfícies oclusais e proximais. Revista Gaúcha Odontologia. Porto Alegre, v.59, n.1, p.117-123, jan./mar. 2011.

2. ASPIRAS M, STOODLEY P, NISTICO $L$ et al. Clinical implications of power toothbrushing on fluoride delivery: effects on biofilm plaque metabolism and physiology. International Journal of Dentistry. Snoqualmie, v.2010, n.651869, p.1-7, apr. 2015.

3. BRASIL. SESC, portal. Odontologia. Disponível em: < http://www.sesc.com.br/portal/saude/odontologia/>. Acesso em: 20 de Abril. 2015, 9:30:00.

4. BRASIL. Projeto SBBrasil 2010: Pesquisa nacional de saúde bucal. Disponível em: <http://dab.saude.gov.br/CNSB/sbbrasil/arquivos/projeto_sb2010_relatorio_final.pdf>Acesso em 14 de Novembro de 2016, $12: 34$.

5. BYEON S.M, LEE MH, BAE TS. The effect of different fluoride application methods on the remineralization of initial carious lesions. Restorative Dentistry \& Endodontics. Jeonju, Korea v. 2016, n.41, p.121-129, may. 2016.

6. CAMPOS ASC, FONSECA L, JUIZ PJL. Antimicrobial activity commercial dentifrice on Streptococcus mutans: in vitro study. Revista Brasileira de Pesquisa em Saúde. Vitória, v.2011, n.13(1), p. 4-10, 2011.

7. CARNEIRO FC, NADANOVSKY P. Dentística Ultraconservativa: fundamentos e técnicas do tratamento da cárie em dentina. 1. ed. São Paulo: Santos, 2003.

8. CONCEIÇÃO EN, LEITE CV. Dentística: Saúde e Estética. 2. ed. Porto Alegre: Artmed Editora, p.596, 2007.

9. COSTA, E, DOMINGUES J, FERREIRA JC et al. Tratamento Medicamentoso de Lesões Iniciais de Cárie: Agentes terapêuticos remineralizantes. Revista Portuguesa de Estomatologia, Medicina Dentária e Cirurgia Maxiloácial, Porto, v. 50, n. 1, p. 43-51, 2009.

10. CURY JA Uso do flúor e controle da cárie como doença. Odontologia Restauradora - Fundamentos e Possibilidades. São Paulo: Editora Santos, cap.2, p. 31-68. Disponível em: <http://w2.fop.unicamp.br/dcf/bioquimica/downloads/mat_consulta4usofluorcontrolecarie.pdf> Acesso em: 29.jul.2016.

11. DAMLE SG, BECTOR A, DAMLE D et al. Effect of dentifrices on their remineralizing potential in artificial carious lesions: An in situ study. Dental Research Journal. India, n. 13, p. 74-79, jan/feb. 2016. 
12. DANTAS A. Cárie dental no Espírito Santo: perspectivas para o controle e prevenção. Revista Brasileira de Pesquisa em Saúde/Brazilian Journal of Health Research, v. 1, n. 1, 2015.

13. FEJERSKOV O, KIDD E. Cárie Dentária: A doença e seu tratamento clínico. 2. ed. São Paulo: Editora Santos, 2005. p. 168.

14. FONTANA M, YOUNG DA, WOLFF MS. Evidence-based caries, risk assessment, and treatment. Dental Clinics of North America. Indianopolis, v.53, n.1, p.149-61, 2009.

15. FONTANA M, ZERO DT. Assessing patients' caries risk. The Journal of The American Dental Association. Indianapolis, n.137, v.9, p.1231-1239, 2006.

16. GUIRADO TE, MENDES FM, BENGTSON AL et al. Contagem de Streptococcus mutans do tecido cariado de dentes decíduos e permanentes. Revista da Associação Paulista de Cirurgiões Dentistas. São Paulo, v.60, n.3, p. 212-217, 2006.

17. HESSE D, BONIFÁCIO CC, MENDES FM et al. Sealing versus partial caries removal in primary molars: a randomized clinical trial. BMC Oral Health. London, v. 14, n.58. , p.14-58, may. 2014.

18. IIJIMA Y. Early detection of white spot lesions with digital camera and remineralization therapy. Australian Dental Journal. Japan, v.53, n.3, p.274-280, 2008

19. KANDUTI D, STERBENK P, ARTNIK B. Fluoride: a review of use and effects on health. Mater Sociomed. Slovenia, v. 28, n. 2, p. 133-137, 2016.

20. KIDD EAM, NYVAB B. Controle da cárie dentária para cada paciente. In: Fejerskov O, Kidd E. Cárie dentária- A doença e seu tratamento clínico. São Paulo: Santos, v.2, p. 303-3011, 2011.

21. LIMA JEO. Cárie dentária: um novo conceito. Revista Dental Press de Ortodontia e Ortopedia Facial, Maringá, v. 12, n. 6, p. 119130, nov./dez. 2007.

22. MALTZ M, JARDIM JJ, ALVES LS. Promotion and dental caries. Brazilian Oral Research. São Paulo, v. 24, n. 1, p. 18-25. 2010.

23. MENDES NS. Avaliação de lesões brancas de cáries: um estudo de prognostico. 2011. 45 f. Dissertação (Mestrado em Odontologia Preventiva e Social; Periodontia e Prótese Dentaria) - Universidade Federal do Rio Grande do Norte, Natal, 2011.

24. MOIMAZ SAS, SALIBA NA, SALIBA O et al. Saúde Bucal e a professora de $1^{\circ}$ grau. Revista Gaúcha de Odontologia. Porto Alegre, V.40, n.4, p. 295-297, 1992.

25. PAULETO ARC, PEREIRA MLT, CYRINO EG. Saúde bucal: uma revisão crítica sobre programações educativas para escolares. Ciência e saúde coletiva, Rio de Janeiro, v. 9, n. 1, p. 121-130, 2004.

26. PERES SHCS, GARCEZ RMVB, PERES AS es et al. Tratamento alternativo de controle da cárie dentária no período maternoinfantil. Revista da Associação Paulista de Cirurgiões Dentistas, São Paulo, v. 55, n. 5, p. 346-350, set./out. 2001.

27. RESTREPO M, BUSSANELI DG, JEREMIAS F et al. Control of White Spot Lesions with Use of Fluoride Varnish or Chlorhexidine Gel During Orthodontic Treatment A Randomized Clinical Trial. Journal of Clinical Pediatric Dentistry.São Paulo, v.40, n.4, p.274-280, 2016.

28. RHIS LB, SOUSA LLGM, WADA RS et al. Carie dentaria segundo nível socioeconômico em Itapetininga /SP/ Brasil. Revista da Faculdade de Odontologia UPF, Passo Fundo, v. 10, n. 2, p. 16-20, jul./dez. 2005

29. ROZIER GR. Effectiveness of methods used by dental professionals for the primary prevention of dental caries. Journal of Dental Education. Washington, v. 65, n. 10, p. 1063-1072, Oct. 2001.

30. SKIEI MS, RAADAL M, STRAND GV et al. The relationship between caries in the primary dentition at 5 years of age and permanente dentition at 10 years of age - a longitudinal study. International Journal of Paediatric Dentistry. Norway. v. 16, n. 3, p. 152 - 160, may. 2006.

31. SOARES JMP, VALENCA AMG. Avaliação clínica do potencial terapêutico do gel e verniz fluoretado na remineralização de lesões cariosas incipientes. Pesquisa Brasileira de Odontopediatria Clinica e Integrada. João Pessoa, v. 3, n. 2, p. 35 - 41, Jul./Dez. 2003.

32. TAGLIAFERRO EPS, AMBROSIANO GMB, MENEGHIM MC et al. Risk indicators na risk predictors of dental caries in schoolchildren. Journal of Oral Applied Science. Bauru, v. 16, n. 6, p. 408-413, dec. 2008.

33. TENUTA LMA, CURY JA. Fluoreto: da ciência à prática clínica. In: Odontopediatria: bases científicas para a prática clínica. São Paulo: Artes Médicas, 2005. p. 113-152.

34. WANG Y, MEI L, GONG $L$ et al. Remineralization of early enamel caries lesions using different bioactive elements containing toothpastes: An in vitro study. Technol Health Care. China, v.14, n.5, p. 701-711, sep. 2016. 